\title{
Jefferson.
}

JHN Journal

Volume 10 | Issue 2

Article 1

Fall 10-1-2015

\section{Fully Endoscopic Microvascular Decompression for Trigeminal Neuralgia}

Christopher J Farrell, MD

Thomas Jefferson University, christopher.farrell@jefferson.edu

Hyunwoo Do, MD

Thomas Jefferson University, hyunwoo.do@jefferson.edu

Sonia Geschwindt, MD

Thomas Jefferson University, sonia.geschwindt@jefferson.edu

James J. Evans, MD

Thomas Jefferson University, James.evans@jefferson.edu

Follow this and additional works at: https://jdc.jefferson.edu/jhnj

Let us know how access to this document benefits you

\section{Recommended Citation}

Farrell, MD, Christopher J; Do, MD, Hyunwoo; Geschwindt, MD, Sonia; and Evans, MD, James J. (2015) "Fully Endoscopic

Microvascular Decompression for Trigeminal Neuralgia," JHN Journal: Vol. 10 : Iss. 2 , Article 1.

DOI: https://doi.org/10.29046/JHNJ.010.2.001

Available at: https://jdc.jefferson.edu/jhnj/vol10/iss2/1

This Article is brought to you for free and open access by the Jefferson Digital Commons. The Jefferson Digital Commons is a service of Thomas Jefferson University's Center for Teaching and Learning (CTL). The Commons is a showcase for Jefferson books and journals, peer-reviewed scholarly publications, unique historical collections from the University archives, and teaching tools. The Jefferson Digital Commons allows researchers and interested readers anywhere in the world to learn about and keep up to date with Jefferson scholarship. This article has been accepted for inclusion in JHN Journal by an authorized administrator of the Jefferson Digital Commons. For more information, please contact: JeffersonDigitalCommons@jefferson.edu. 


\section{Fully Endoscopic Microvascular Decompression for Trigeminal Neuralgia}

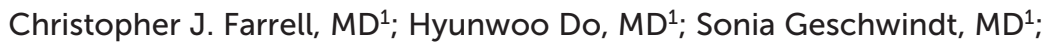 \\ James J. Evans, $M D^{1,2}$ \\ 1Department of Neurological Surgery, Thomas Jefferson University, Philadelphia, PA \\ 2Department of Otolaryngology, Thomas Jefferson University, Philadelphia, PA
}

Trigeminal neuralgia (TN) is a chronic, progressive facial pain disorder characterized by severe paroxysmal episodes in the distribution of the trigeminal nerve. The most common cause of (TN) is compression of the trigeminal nerve by a vascular structure within the posterior fossa at the dorsal root entry zone (DREZ). Initially described by Dr. Peter Janetta, microvascular decompression has been clearly demonstrated to be a safe and effective treatment for TN with excellent immediate and long-term pain relief. ${ }^{1}$

Although neuroimaging has advanced significantly allowing for improved pre-operative visualization of the trigeminal nerve and determination of vascular conflict, most neurosurgeons continue to practice the MVD procedure in a very similar manner to Dr. Janetta's 1967 description. ${ }^{2}$ While the retrosigmoid craniotomy and operative microscope allows for an excellent view of the posterior aspect of the trigeminal nerve within the cerebellopontine angle, visualization of the anterior aspect of the nerve is limited. Additionally, adequate visualization of the DREZ may be difficult and require additional retraction of the cerebellum, potentially resulting in complications such as hearing loss and cerebellar injury. As neurosurgical experience with the endoscope has grown, a variety of authors have described performing microvascular decompression with endoscopic assistance which involves using the endoscope to inspect the trigeminal nerve for sites of compression but performing the decompression under the microscope. While the main advantage of the endoscopic approach compared to the microscopic approach is improved visualization of the trigeminal nerve from the DREZ to Meckel's cave including its inferior, anterior and superior surfaces, evolution of the procedure to a fully endoscopic approach has the additional benefits of being less invasive with minimal soft tissue dissection and cerebellar retraction allowing for reduced patient discomfort and accelerated recovery. In this technical review, we describe our approach to performing a fully endoscopic microvascular decompression including the surgical nuances that allow the procedure to be performed safely and efficiently.

\section{SURGICAL PLANNING AND INSTRUMENTATION}

The indications for the fully endoscopic MVD do not differ from those of the microscopic approach. However surgical instrumentation varies considerably beyond use of the endoscope. While the asterion represents an
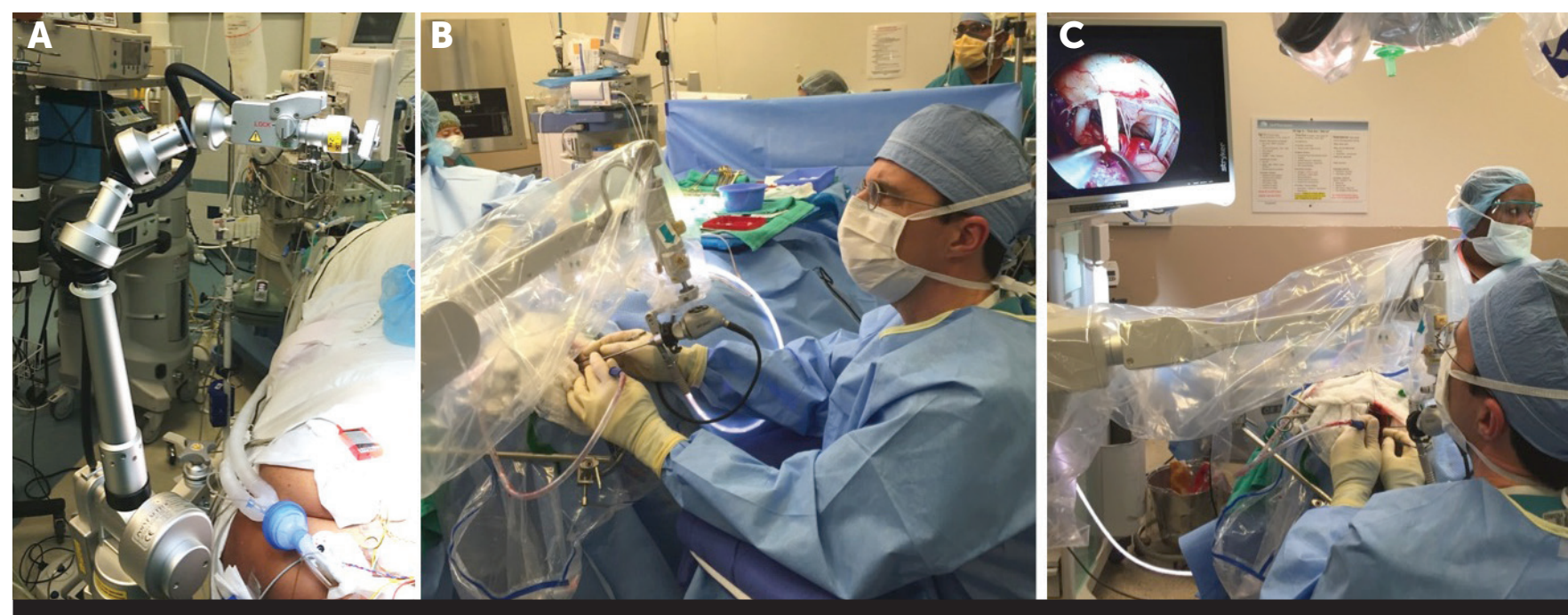

Figure 1

A. The pneumatic endoscopic holding arm is fixed to the contralateral side of the OR table prior to draping. B. The holding arm and $2.4 \mathrm{~mm}$ endoscope are positioned parallel to the posterior petrous ridge. $\mathbf{C}$. The endoscope is placed along the tentorium and fixed proximal to the internal auditory canal allowing instruments to be brought into the field above and below the endoscope. 

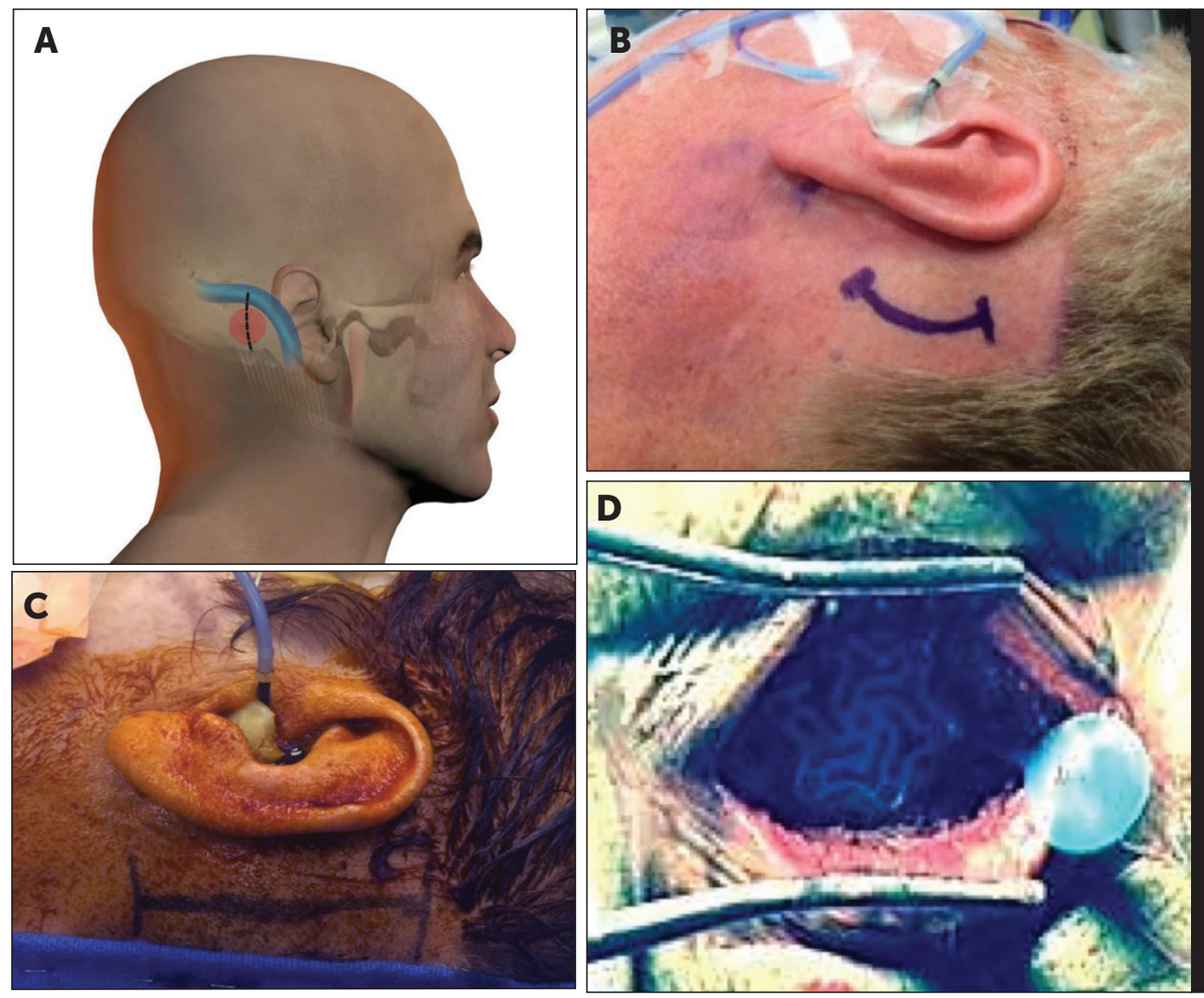

Figure 2

A. Illustration of place-

ment of the incision

(dotted line) and bony

opening (shaded area)

relative to the transverse

and sigmoid sinuses. B.

Slightly curvilinear $2 \mathrm{~cm}$

incision is planned for

endoscopic MVD, which

is significantly reduced in

length compared to our

standard microscopic

MVD incision (C). D. The

bony opening for the

endoscopic MVD is made

to be the same size as a

$14 \mathrm{~mm}$ burr-hole cover.

important landmark for any retrosigmoid craniotomy, we prefer to also utilize image-guidance during the fully endoscopic approach as accurate placement of the incision is critical to perform the procedure is a truly minimally-invasive manner. Additionally, we utilize bipolars (SILVERGlide Bipolar Forceps, Stryker, Kalamazoo MI) with ultra-thin tines of variable lengths that enable increased maneuverability when working through small craniotomies and bayonetted, rotating microdissectors (Evans Rotatable instruments, Mizuho America Inc., Union City, CA) designed for endoscopic use. Although dynamic endoscopy is extremely helpful in establishing depth of field and is the preferred technique for endonasal endoscopy, the restricted anatomy of the cerebellopontine angle lends itself better to fixed endoscopy. Although several endoscopic holders are commercially available, we have utilized the Mitaka holding arm (Mitaka Kohki Co., Tokyo, Japan) which is OR-table mounted with multiple joints and pneumatically controlled, allowing for one-handed manipulation (Figure 1). We typically begin the procedure using a $4 \mathrm{~mm}, 0$-degree rigid endoscope (Karl Storz, Tuttlingen, Germany) that is attached to the holder arm. However, if access is limited, we not infrequently utilize a pediatric $2.4 \mathrm{~mm}$ rigid endoscope to increase our working area for bimanual dissection. Angled endoscopes (30-degree, 70-degree) are employed as necessary. A high-definition camera and monitor are critical for performance of the fully endoscopic approach as safe dissection of the arachnoid is contingent upon subtle visual cues. Although obscuration of the endscope lens may occur, we do not utilize an endoscope lens cleaner during this procedure as this device increases the circumference of the endoscope and further restricts working area. We have found that gentle intermittent irrigation of the endoscope by the assistant effectively restores image quality.

\section{INCISION AND CRANIOTOMY}

We typically perform an approximately $2 \mathrm{~cm}$ linear or curvilinear retroauricular incision enabling placement of a $14 \mathrm{~mm}$ diameter burr hole-type craniectomy at the sigmoid-transverse junction (Figure 2). In patients with thicker skin and musculature, the curvilinear incision is preferred as the slight posterior extension does not allow the soft tissue and self-retaining retractor to restrict the angle of the endoscope along the posterior petrous bone. If instrumentation optimized for endoscopic utilization is not available, we recommend using a slightly increased craniectomy size of approximately $18 \mathrm{~mm}$. Minimal muscular disruption is performed due to the more superior location of the craniotomy and a self-retaining retractor or stay-sutures can be used to retract the skin edges. The burr hole is drilled with a $6 \mathrm{~mm}$-round cutting bit providing approximately $2 \mathrm{~mm}$ of exposure of the inferior aspect of the transverse sinus and posterior aspect of 


\section{Figure 3}

A. Illustration of the positioning of the endoscope proximal to the cranial nerve VII/VIII complex (*), allowing for view of the trigeminal nerve root entry zone and sites of potential vascular compression (arrows) B. Endoscopic view of trigeminal nerve and site of vascular compression at nerve root entry zone (arrow).
A
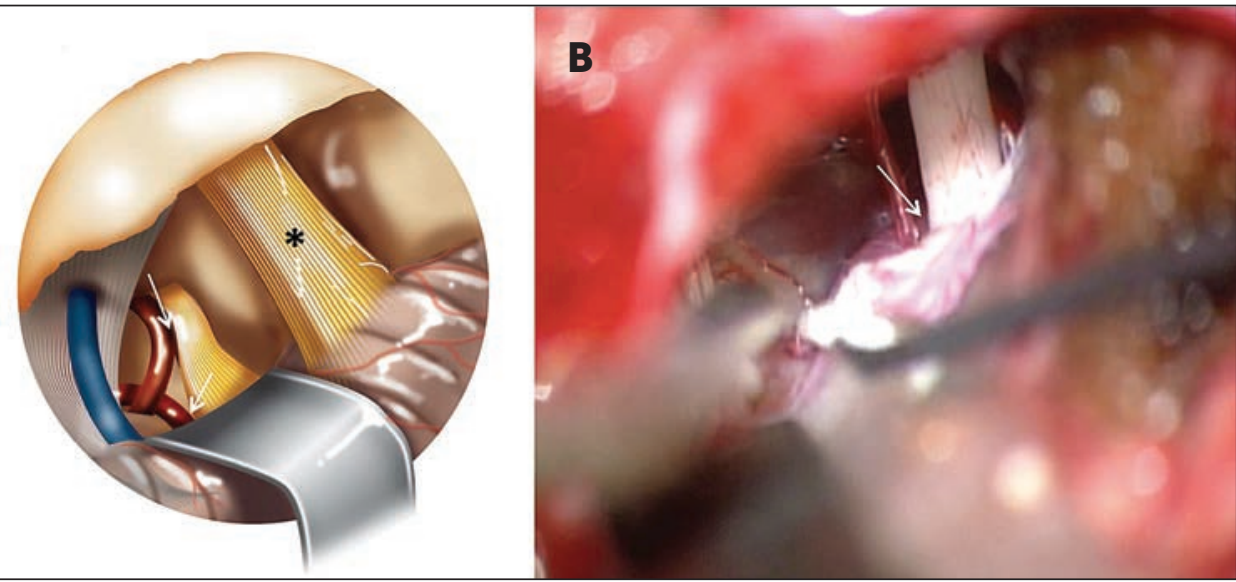

\section{Figure 4}

A. Illustration of placement of PTFE (Teflon) pledget $(*)$ between trigeminal nerve and compressing artery B. Endoscopic view with bimanual manipulation of pledget.
A

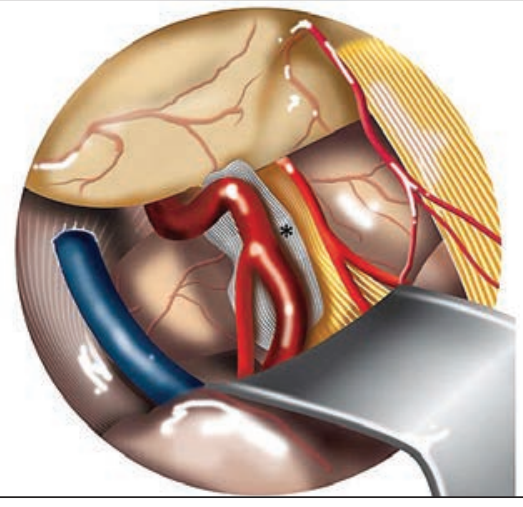

B

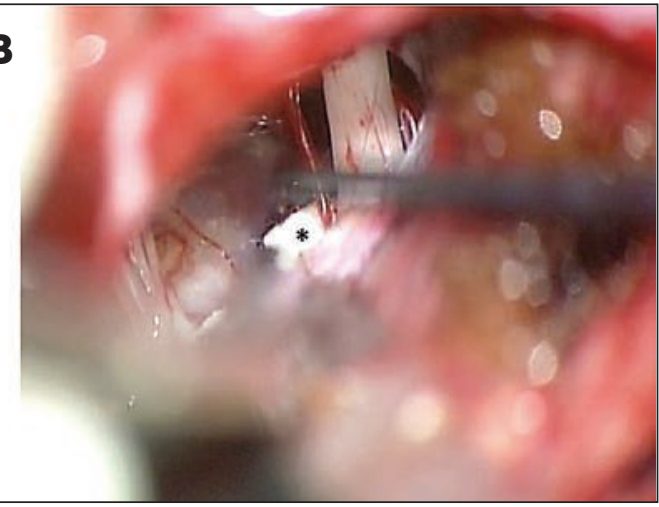

the sigmoid sinus. Failure to adequately remove bone over the sinuses will limit the final positioning of the endoscope and may necessitate increased cerebellar retraction.

\section{EXPOSURE AND DECOMPRESSION OF THE TRIGEMINAL NERVE}

The dura is then opened in a C-shaped fashion extending from the transverse sinus edge to the sigmoid sinus edge followed by a bisection of the dura toward the sigmoid-transverse sinus junction. The dural leaflets are then retracted with stay-sutures. Similar to the skin incision, the posterior opening of the dura over the cerebellum allows the endoscope to be inserted with increased degree of freedom and to achieve the optimal angle for visualization and instrument maneuverability. The supero-lateral aspect of the cerebellum is then gently retracted and the endoscope advanced into the cerebellopontine angle along the tentorium under endoscopic visualization.
As access to the cisterna magna is not possible, temporary placement of a fixed retractor may be necessary at this point to allow for the arachnoid above the cranial nerve $7 / 8$ complex to be sharply dissected and cerebrospinal fluid gently aspirated to facilitate cerebellar relaxation. The trigeminal nerve is then inspected from the DREZ to Meckel's cave for any sights of vascular compression with the 0 -degree and angled endoscopes dynamically (Figure 3). At this point, the fixed cerebellar retractor is removed and the endoscope repositioned to its optimal location, typically along the tentorial edge allowing for instruments to be passed more inferiorly. No further cerebellar retraction is necessary throughout the procedure, although sacrifice of the superior petrosal vein is frequently necessary to achieve optimal positioning of the endoscope. Placement of the endoscope along the posterior petrous face allows for an excellent view of the inferior aspect of the trigeminal nerve but requires a more oblique angulation of the endoscope that restricts maneuverability. Additionally, the endoscopic view may be compromised by a prominent suprameatal tubercle. The lateral cerebellar surface is covered with a rubber dam to allow for easy repetitive introduction of instruments without incurring cerebellar surface injury. Although cottonoids can serve a similar function, their thickness can prove obtrusive during this minimally invasive approach. Similar to the microscopic approach, bimanual manipulation of the trigeminal nerve and offending artery is then performed with buffering of the nerve with a small piece of polytetrafluoroethylene (Figure 4). Once the decompression has been performed, circumferential inspection of the trigeminal nerve should be repeated with angled endoscopes to ensure no further areas of compromise, including the DREZ, prior to endoscope removal.

\section{DURAL AND BONY RECONSTRUCTION}

Similar to the microscopic approach, careful attention to dural closure is 
important to prevent post-operative cerebrospinal fluid leakage, however, with the endoscopic approach, the dura can typically be repaired primarily as there is no thermal injury to the dura. The craniectomy site is inspected for any air cells and waxed appropriately followed by reconstruction with a $14 \mathrm{~mm}$ titanium burr hole cover plate (Figure 1).

\section{REFERENCES}

1. Barker FG, Jannetta PJ, Bissonette DJ, Larkings MV, Jho HD. The Long-Term Outcome of Microvascular Decompression for Trigeminal Neuralgia. NEJM. 1996; 334: 1077-1083

2. Jannetta PJ. Arterial Compression of the Trigeminal Nerve at the Pons in Patients with Trigeminal Neuralgia. J Neurosurg. 1967. 26: 159-162. 